\title{
CONCEPÇÃO DE SEXUALIDADE ENTRE PESSOAS COM DEFICIËNCIA VISUAL
}

\author{
CONCEPTION OF SEXUALITY AMONG PEOPLE WITH VISUAL DISABILITIES
}

\author{
Mariana Crisci Cozac \\ Departamento de Fisioterapia Aplicada \\ Instituto de Ciências da Saúde - ICS \\ Universidade Federal do Triângulo Mineiro - UFTM
}

Andrea Ruzzi Pereira

Departamento de Terapia Ocupacional; ICS; UFTM

\section{Shamyr Sulyvan de Castro}

Departamento de Fisioterapia

Universidade Federal do Ceará - UFC

\author{
Contato \\ Shamyr Sulyvan de Castro \\ Rua José Vilar, 520, apto 801 , Meireles \\ Fortaleza - CE \\ CEP: $60125-000$ \\ E-mail: shamyrsulyvan@gmail.com
}

Pesquisa desenvolvida no

Departamento de Fisioterapia Aplicada da UFTM

Bolsa concedida: PIBIC/CNPq

\section{RESUMO}

O artigo buscou descrever as concepções de pessoas cegas sobre sexualidade, mais especificamente sobre os temas: relações sexuais, ficar e namorar; e verificar a existência de diferentes discursos de acordo com o estado civil dos sujeitos. Trata-se de um estudo qualitativo, transversal de caráter descritivo, realizado no Instituto de Cegos do Brasil Central ICBC, situado na cidade de Uberaba - MG. A amostra final foi composta de 51 pessoas, sendo 26 do sexo masculino que passaram por entrevistas com questionários específicos, no período de agosto de 2012 a abril de 2013. Essas entrevistas foram gravadas e transcritas para posteriormente serem analisadas pelo Discurso do Sujeito Coletivo. Foi observado que as ideias das pessoas unidas se mostraram mais

conservadoras do que as dos não unidos, no entanto, as ideias reforçadas pela mídia são aquelas que mais se aproximam do diálogo dos jovens solteiros; fato este que pode ser conferido ao estado conjugal e à idade. Isso pode ser atribuído à íntima relação entre a necessidade de relação sexual quando se fala sobre ficar para as pessoas não unidas, enquanto para os unidos o namoro é mais importante e duradouro, pois é dele que poderá surgir o casamente entre as pessoas. Cabe, portanto, ao profissional da saúde que está sempre em contato com essa população esclarecer as dúvidas e proporcionar informações corretas sobre a sexualidade, para que, assim, possam ter elementos para uma correta abordagem do tema, buscando sempre a preservação da saúde. Palavras-chave: Sexualidade. Transtornos da visão. Saúde Sexual. 


\section{ABSTRACT}

The article aimed to describe the conception of blind people concerning sexuality, specifically on the topics: sexual intercourse, hooking up and dating; and to check for different discourses according to the marital status of individuals. This is a cross-sectional descriptive qualitative study, held at the Institute for the Blind of Central Brazil - ICBC, located in Uberaba - MG. The final sample consisted of 51 people, including 26 males who have undergone interviews with specific questionnaires, between August 2012 and April 2013. The interviews were recorded and transcribed to be later analyzed by the Collective Subject Discourse. It was observed that the ideas of people in a relationship were more conservative than that of single people, but the ideas reinforced by the media are those are closest to the dialogue of young single people. This fact can be attributed to marital status and age. This can be attributed to the close relationship between the need for intercourse when one talks about hooking up to single people, while people in a relationship dating is more important and enduring because it is from that that a marriage may result. Therefore, it is up to the health professional who is always in contact with that population to answer questions and provide correct information about sexuality, so that they can have elements for a correct approach to the subject, always seeking the preservation of health.

Keywords: Sexuality. Blindness. Sexual health.

\section{INTRODUÇÃO}

egalmente, a deficiência visual é definida por dois decretos, o de $n^{\circ} 3.298 / 99^{1}$ e o de $n^{\circ} 5.296 / 04^{2}$, conceituando-se como deficiência visual: cegueira - na qual a acuidade visual é igual ou menor que 0,05 no melhor olho, com a melhor correção óptica; baixa visão - acuidade visual entre 0,3 e 0,05 no meIhor olho, com a melhor correção óptica; os casos nos quais a somatória da medida do campo visual em ambos os olhos for igual ou menor que $60^{\circ}$; ou a ocorrência simultânea de quaisquer das condições anteriores.

É sabido que as pessoas com deficiência visual vivenciam diversas situações de exclusão, sendo ela social ${ }^{3}$, educacional ${ }^{4}$, na saúde ${ }^{5}$, entre outras. Essa exclusão se deve à própria deficiência ou às dificuldades do meio em que vivem e realizam suas atividades cotidianas.

As pessoas com cegueira enfrentam obstáculos ao expressar sua sexualidade, especialmente por causa do preconceito, manifestado de diversas formas, e da falta de informação sobre a sua sexualida$\mathrm{de}^{6}$. Para conhecer e relacionar-se consigo mesmo, com o outro e com o meio, a pessoa com deficiência visual precisa vivenciar experiências corporais que permitam essas descobertas ${ }^{7}$. No entanto, há uma percepção positiva da sexualidade, compreendendo-a como uma manifestação natural do ser humano, que envolve doação, intimidade, afirmação da orientação sexual, que podem propiciar experiências positivas na vida, bem como o matrimônio ${ }^{6}$.

As pessoas com deficiência visual apresentam o mesmo desenvolvimento afetivo-sexual que pessoas videntes; querem conhecer seus corpos e o respectivo funcionamento, o que as faz explorar o ficar e o namorar antes do casamento como qualquer pessoa. No entanto, essa curiosidade por parte da pessoa com cegueira nem sempre é bem-vista pelos outros, o que caracteriza a visão conservadora mantida por alguns ${ }^{8}$.
Cabe ainda ressaltar que a sexualidade é discutida não somente para as pessoas com acometimentos visuais, mas também para outros casos de ocorrência de deficiência, entre elas a intelectual ${ }^{9,10}$, física ${ }^{11}$ e surde $z^{12}$.

Consideradas tabu em nossa sociedade em decorrência da estruturação cultural, questões relacionadas com a sexualidade são um ponto de difícil discussão entre a maioria das pessoas, agravando-se as dificuldades quando o assunto se refere à pessoa com qualquer deficiência, não sendo diferente às pessoas com deficiência visual. Portanto, conhecer as percepções das pessoas com deficiência visual acerca de sua sexualidade oportuniza verificar a necessidade de informações e conhecimentos com relação às questões que envolvem a sexualidade.

Trata-se de uma população vítima de exclusão social ${ }^{13}$ e que sabidamente necessita de recursos diferenciados para o processo de aprendizagem ${ }^{14,15}$. Por essa razão, expor o ponto de vista dos deficientes visuais permitirá à sociedade conhecer quais são as dúvidas mais frequentes ou os problemas que eles vêm enfrentando, buscando a facilitação do trabalho dos profissionais de saúde e a elaboração de estratégias úteis que promovam a oferta de informações corretas e necessárias para o exercício saudável da sexualidade, em linguagem adequada. Além disso, pode oferecer subsídios para o planejamento em saúde ou criação de programas e/ou políticas de saúde direcionadas a esse grupo populacional. Nesse sentido, o domínio dessas informações por profissionais da área de educação também pode ter sua utilidade à medida que o tema poderá ser tratado de forma mais adequada pelos educadores.

Desse modo, esta pesquisa teve como objetivo principal descrever as concepções de pessoas cegas sobre sexualidade, mais especificamente sobre os temas: relações sexuais, ficar e namorar. Secundariamente, pretendeu-se verificar se existem diferentes discursos de acordo com o estado civil dos sujeitos. 
MÉTODO

$\mathrm{T}$ rata-se de um estudo qualitativo, transversal de caráter descritivo, realizado no Instituto de Cegos Brasil Central - ICBC, situado na cidade de Uberaba - MG, que atendia, à época da pesquisa, 160 pessoas com algum tipo de deficiência visual associada ou não a outras deficiências. O ICBC é uma entidade do terceiro setor, fundada em 1942, que presta assistência na forma de atendimento em saúde, escola fundamental, apoio educacional à rede regular de ensino, transcrição, adaptação e confecção de livros e materiais pedagógicos para deficientes visuais, avaliação funcional da visão, estimulação visual, informática adaptada, cursos de capacitação para profissionais na área da deficiência visual, orientação e mobilidade, estimulação essencial, estimulação sensorial, atividades de vida diária, datilografia e escrita Braille, capacitação e inserção de deficientes visuais no mercado de trabalho, oficina pedagógica, artesanato e sala de recursos multifuncionais ${ }^{1}$.

Para este estudo, foram sorteados aleatoriamente $50 \%$ da população atendida mantendo a paridade entre ambos os sexos, de modo que foram selecionadas 70 pessoas. Como fator de exclusão, considerou-se a ocorrência de deficit cognitivo indicado por registro médico. Por esse critério, foram excluídas 50 pessoas, que foram repostas também por sorteio aleatório. Recusaram-se a participar dessa pesquisa 10 mulheres e 9 homens. Portanto, a amostra final foi composta de 51 pessoas, sendo 26 do sexo masculino, com idade máxima de 84 e mínima de 16 anos (média 41,83; DP: 20,63); e 25 muIheres com idade entre 73 e 17 anos, respectivamente entre os homens (média 40; DP: 19,01).
Para esta pesquisa, foi criado um questionário semiestruturado abrangendo inclusive os temas aqui estudados. As perguntas contidas no questionário foram as seguintes: "Explique o mais detalhadamente possível o que você sabe sobre as relações sexuais"; "Explique o mais detalhadamente possível o que você sabe sobre: a) ter relações sexuais b) namorar c) ficar".

As entrevistas foram realizadas individualmente, com todos os sujeitos selecionados, em um local reservado no próprio ICBC ou na casa dos indivíduos (dois casos), da forma como eles preferissem e gravadas para posterior transcrição. Os sujeitos não tinham tempo determinado para responder às questões e só aderiam à pesquisa caso fosse de sua vontade. A coleta de dados aconteceu de agosto de 2012 a abril de 2013, e as entrevistas foram realizadas por uma pesquisadora treinada para essa atividade.

Após leitura do Termo de Consentimento Livre Esclarecido pelo entrevistador ou pelo entrevistado na versão em Braille, a resposta para a adesão à pesquisa era dada verbalmente e gravada. No caso dos menores de 18 anos, o assentimento era dado pelo menor e o consentimento pelos responsáveis. Feito isso, iniciavam-se as demais perguntas, como as citadas anteriormente, e um breve e simples questionário socioeconômico, que abrangia variáveis como idade, escolaridade, renda, filhos, relações sexuais e sua frequência da prática.

As gravações das respostas ao questionário qualitativo foram transcritas na íntegra, e o conteúdo foi analisado segundo o método do Discurso do Sujeito Coletivo $(\mathrm{DSC})^{16}$, possibilitando a seleção das ideias centrais de todas as respostas.

\section{RESULTADOS E DISCUSSÃO}

Amostradas na tabela 1 .

TABELA 1. Descrição da amostra segundo variáveis estudadas

\begin{tabular}{|c|c|c|c|c|c|c|}
\hline \multirow[t]{2}{*}{ Variável } & \multicolumn{2}{|c|}{ Masculino } & \multicolumn{2}{|c|}{ Feminino } & \multicolumn{2}{|c|}{ Total } \\
\hline & $\mathbf{n}$ & $\%$ & $\mathbf{n}$ & $\%$ & n & $\%$ \\
\hline \multicolumn{7}{|l|}{ Escolaridade } \\
\hline Analfabeto & 6 & 25 & 2 & 7,41 & 8 & 15,68 \\
\hline Básico incompleto & 3 & 12,50 & 0 & 0 & 3 & 5,88 \\
\hline Básico completo & 2 & 8,40 & 0 & 0 & 2 & 3,93 \\
\hline Médio incompleto & 3 & 12,50 & 8 & 29,63 & 11 & 21,56 \\
\hline Médio completo & 4 & 16,60 & 4 & 14,82 & 8 & 15,68 \\
\hline Superior incompleto & 1 & 4,16 & 3 & 11,11 & 4 & 7,84 \\
\hline Superior completo & 5 & 20,84 & 10 & 17,03 & 15 & 29,43 \\
\hline \multicolumn{7}{|l|}{ Estado civil } \\
\hline Solteiro & 17 & 65,39 & 14 & 56,00 & 31 & 60,78 \\
\hline Casado/unido & 9 & 34,61 & 7 & 28,00 & 16 & 31,38 \\
\hline Viúvo & 0 & 0,00 & 4 & 16,00 & 4 & 7,84 \\
\hline Filhos & 10 & 38,46 & 8 & 32,00 & 18 & 35,29 \\
\hline Relações sexuais & 24 & 92,30 & 17 & 68,00 & 41 & 80,39 \\
\hline
\end{tabular}

Fonte: os autores, 2014 
A renda média dos entrevistados era de aproximadamente R\$2.000 (DP 1316,91) entre as mulheres; de aproximadamente $\mathrm{R} \$ 1.500$ (DP 847,71) entre os homens e de $\mathrm{R} \$ 1.700$ (DP 1123,04) para toda a amostra. A porcentagem de pessoas que relataram ter relações sexuais com frequência entre os entrevistados foi de $53,84 \%$ entre os homens e de $25,39 \%$ entre as mulheres.

Pela análise dos dados coletados, foram encontradas 25 ideias centrais para a primeira questão e 39 para a segunda. Feito isso, elas foram agrupadas de forma crescente, considerando a frequência de ocorrência para cada uma das questões. Na tabela 2 são apresentadas as ideias centrais mais frequentes para cada questão.

TABELA 2. Distribuição das ideias centrais mais frequentes encontradas para cada uma das questões segundo estado civil

\begin{tabular}{|c|c|c|c|c|}
\hline Ideias centrais & $\begin{array}{l}\text { Viúvo } \\
\text { n(\%) }\end{array}$ & $\begin{array}{l}\text { Unido } \\
\mathrm{n}(\%)\end{array}$ & $\begin{array}{l}\text { Solteiro } \\
\mathbf{n}(\%)\end{array}$ & $\begin{array}{l}\text { Total } \\
\mathbf{n}(\%)\end{array}$ \\
\hline \multicolumn{5}{|l|}{$\begin{array}{l}\text { Explique o mais detalhadamente possível o que você } \\
\text { sabe sobre relações sexuais }\end{array}$} \\
\hline Relação sexual ocorre apenas com o & $2(33,33)$ & $2(7,14)$ & $10(15,39)$ & $14(12,63)$ \\
\hline \multicolumn{5}{|l|}{ companheiro/parceiro entre pessoas com o sexo oposto } \\
\hline $\begin{array}{l}\text { Relação sexual acontece entre duas pessoas dentro dos } \\
\text { limites, costumes e desejos de cada um }\end{array}$ & $0(0)$ & $5(17,85)$ & $6(9,23)$ & $11(11,57)$ \\
\hline Relação sexual serve para reprodução & $0(0)$ & $3(10,73)$ & $7(10,76)$ & $10(10,52)$ \\
\hline \multicolumn{5}{|l|}{$\begin{array}{l}\text { Explique o mais detalhadamente possível o que você } \\
\text { entende por ficar }\end{array}$} \\
\hline Ficar é algo descompromissado, que acontece entre duas & $1(20)$ & $4(28,60)$ & $17(37,77)$ & $22(34,37)$ \\
\hline \multicolumn{5}{|l|}{ pessoas e que não necessariamente vá durar mais tempo } \\
\hline Ficar envolve relação sexual com prazer & $2(40)$ & $1(7,14)$ & $11(24,45)$ & $14(21,87)$ \\
\hline Termo que permite muita liberdade & $1(20)$ & $0(0)$ & $2(4,45)$ & $3(4,68)$ \\
\hline Eu não concordo com isso & $0(0)$ & $1(7,14)$ & $2(4,45)$ & $3(4,68)$ \\
\hline
\end{tabular}

Explique o mais detalhadamente possível o que você entende por namorar

Pode resultar em um casamento ou não, é uma fase de compartilhar, mas é uma coisa nova, poder passar por essa fase

Envolve respeito, consideração e carinho de ambas as partes, sempre dentro de seus limites

É um relacionamento mais sério $14(24,14)$ $16(23,18)$

Explique o mais detalhadamente possível o que você entende por ter relações sexuais

Para ter relação tem que ter sentimento

Acontece entre dois parceiros que se sentem seguros e são fieis com prevenção

Ter prazer e dar prazer $1(16,67)$ $0(0)$ $10(32,75)$ $20(28,98)$

Necessidade

$\begin{array}{llll}1(16,67) & 2(11,11) & 9(20,00) & 12(17,39) \\ 0(0) & 6(33,33) & 6(13,33) & 12(17,39) \\ 0(0) & 5(27,77) & 5(11,12) & 10(14,49) \\ 1(16,67) & 2(11,11) & 7(15,56) & 10(14,49)\end{array}$


Para a maioria dos participantes, a relação sexual deve acontecer prioritariamente entre duas pessoas do sexo oposto, que se gostam e que devem respeitar o limite de cada uma das duas partes, sendo que o maior propósito é a reprodução, por isso é um ato que deve ocorrer entre pessoas que já possuem uma relação matrimonial. Essa ideia central se baseia em um padrão socialmente imposto, associado à rigidez social de estabelecimento de papéis, com dificuldade na mudança de padrões, mesmo quando se pensa na homossexualidade, bem como a relação sexual fora do contexto matrimonial ${ }^{17}$.

Relacionamentos sem compromissos como, por exemplo, o ficar são aprovados majoritariamente por aqueles mais jovens e solteiros, já que se trata de uma relação sem compromissos, que não necessariamente vá durar mais tempo. Além disso, os não unidos afirmam que o ficar deve necessariamente envolver relação sexual, com o intuito de proporcionar prazer um ao outro, sem maiores envolvimentos sentimentais. Essa prática se difere do namorar quando comparada com o tempo de duração, o gostar, o nível de compromisso e seriedade, assim como a confiança e sinceridade, que definem o limite entre o namorar e o ficar ${ }^{8}$.

O namoro é notadamente apoiado pelas pessoas unidas, já que o consideram como uma oportunidade de conhecer melhor a outra pessoa, sendo uma fase de respeito, carinho recíproco dentro dos limites estabelecidos por ambos; é um relacionamento mais sério que o ficar e pode resultar em casamento. Namorar envolve um relacionamento mais fechado e que necessita de uma menor exposição diante da sociedade, muitas vezes essa preferência acontece pelo medo de se expor que essa população apresenta; pelo receio dos obstáculos geralmente enfrentados, principalmente quando relacionado com sua sexualidade ${ }^{6}$.

Ter relação sexual é, para ambos os grupos, uma forma de dar e receber prazer e deve acontecer entre duas pessoas que se sentem seguros entre si e que mantêm uma relação fiel. No entanto, para os não unidos, é visível que a maior parte tem a relação sexual como uma forma de sanar suas necessidades carnais. Essa ideia também foi encontrada em um estudo no qual os jovens relatavam a possibilidade de haver sexo sem amor e vice-versa ${ }^{8}$. O mesmo destaque se dá quando questionados sobre a necessidade de envolver sentimentos, os não unidos julgam mais necessário haver sentimentos entre as partes quando comparados aos unidos. 
Diante os resultados apresentados acima, as ideias centrais se aproximam muito das ideias apresentadas por pessoas sem deficiência em um estudo de 2005 no qual foram descritas opiniões e atitudes sobre sexualidade da população urbana brasileira, em que as pessoas relacionam à prática de relações sexuais majoritariamente com envolvimento e sentimentos ${ }^{18}$.

No que tange à escolaridade, as ideias se diferem no ponto de vista biológico e literário, já que quanto mais anos de estudo, maior era o conhecimento sobre a sexualidade e mais técnicos eram os termos usados. No entanto, mesmo as pessoas com menos anos de estudo não mostraram informações equivocadas, eram apenas populares. Diferentemente do que foi visto no mesmo estudo de 2005 em que as informações foram as mesmas de acordo com a escolaridade ${ }^{18}$.

O fato de as informações obtidas serem muito semelhantes aos populares e aos fornecidos pela mídia pode ser atribuído à baixa escolaridade em alguns casos e também à vergonha de conversar sobre esses assuntos com os familiares. Isso acontece pela profunda dificuldade que os pais têm ante a sexualidade de seus filhos; e, por isso, acabam transferindo esse papel educativo a terceiros, reproduzindo, então, formas disciplinares de controle que perpetuam um ciclo ao longo das gerações ${ }^{8}$. Além disso, essas informações se disseminam entre eles, pois a conversa entre amigos também se faz como fonte de informação sobre esse tipo de assunto, como foi visto em um estudo porto-riquenho de 2001, no qual a conversa com os amigos foi a terceira fonte mais votada para assuntos desse gênero ${ }^{19}$.

Os participantes mostraram-se sempre cientes de tudo o que envolvia sua sexualidade e não hesitaram em responder às questões, o que pode ser atribuído pelo fato de as pessoas com deficiência conhecerem o imaginário social a seu respeito e acreditarem que a divulgação de estudos como este devem desmistificar os conceitos sobre a sexualidade dessa população ${ }^{20}$.

Uma limitação que pode ser considerada ao estudo é o fato de que a entrevistadora de todos os participantes era do sexo feminino. Isso fazia com que alguns entrevistados se sentissem constrangidos em passar algumas informações de modo a omitir dados que poderiam ser importantes. A existência de entrevistadores de ambos os sexos permitiria ao entrevistado escolher com quem gostaria de realizar a entrevista. No entanto, o estudo foi capaz de demonstrar as concepções sexuais de pessoas com deficiência visual, o que abre caminho para aprender como lidar com essa fase de construção da sexualidade delas, tanto no âmbito pessoal quanto emocional.

\section{CONCLUSÕES}

$\mathrm{N}$ o presente estudo, foi observado que as ideias das pessoas unidas se mostraram mais conservadoras do que as dos não unidos, provindas de concepções familiares fortes e bastante aterrorizadas pela mídia; no entanto a ideias reforçadas pela mídia são aquelas que mais se aproximam do diálogo dos jovens solteiros. Fato este que pode ser atribuído ao estado conjugal e à idade.

Isso acontece em decorrência das concepções matrimoniais impostas pela sociedade e que extrema o pensamento dos indivíduos, para pensamentos liberais ou conservadores, que se adequam aos não unidos e aos unidos respectivamente. Isso pode ser notado pela íntima relação entre a necessidade de relação sexual quando falamos sobre ficar para as pessoas não unidas, enquanto para os unidos o namoro é mais importante e duradouro, pois é dele que poderá surgir o casamente.

Por isso, cabe ao profissional da saúde que está sempre em contato com essa população esclarecer as dúvidas e proporcionar informações corretas sobre a sexualidade, para que, assim, possam ter elementos para uma correta abordagem do tema, buscando sempre a preservação da saúde. 


\section{Referências}

1. Brasil P da R. Decreto No 3.298, de 20 de Dezembro de 1999. Brasília; 1999. http://www.planalto.gov.br/ccivil_03/decreto/d3298.htm.

2. Brasil P da R. Decreto No $5.296 \overline{6}$ de 2 de Dezembro de 2004. Brasília; 2004. http://www.planalto.gov.br/ccivil_03/_ato2004-2006/2004/decreto/d5296.htm.

3. Clarke PJ, Ailshire JA, Nieuwenhuijsen ER, de Kleijn - de Vrankrijker MW. Participation among adults with disability: The role of the urban environment. Soc Sci Med. 2011;72(10):1674-1684. doi:10.1016/j.socscimed.2011.03.025.

4. Castro SS, Lefèvre F, Lefèvre AMC, Cesar CLG. Accessibility to health services by persons with disabilities. Rev. Saúde Pública. 2011;45(1):99-105. http://www.ncbi.nlm.nih.gov/pubmed/21049172. Accessed August 4, 2014.

5. Castro SS de, César CLG, Carandina L, Barros MBA, Alves MCGP, Goldbaum M. Deficiência visual, auditiva e física: prevalência e fatores associados em estudo de base populacional. Cad Saude Publica. 2008;24(8):1773-1782. doi:10.1590/S0102-311X2008000800006.

6. França DNO. Sexualidade da pessoa com cegueira: da percepção à expressão. Rev Bras Educ Espec. 2013;19(4):583-596.

7. Nasário JC, Ernst TN. Imagem corporal e a deficiência visual: a educação física na compreensão do corpo. In: X Congresso Nacional de Educação.; 2011:260-274. http://educere.bruc.com.br/CD2011/pdf/4333_2325.pdf.

8. Bezerra CP, Pagliuca LMF. A vivência da sexualidade por adolescentes portadoras de deficiência visual. Rev. da Esc. Enferm. da USP. 2010;44(3):578-583. doi:10.1590/S0080-62342010000300005.

9. Morales AS, Batista CG. Compreensão da sexualidade por jovens com diagnóstico de deficiência intelectual. Psicol Teor e Pesqui. 2010;26(2):235-244. doi:10.1590/S0102-37722010000200005.

10. Bastos OM. Sexualidade e deficiência intelectual: narrativas de pais de adolescentes. Physis Rev. Saúde Coletiva. 2012;22(3):1031-1046. doi:10.1590/S0103-73312012000300010.

11. Berman H, Harris D, Enright R, Gilpin M, Cathers T, Bukovy G. Sexuality and the adolescent with a physical disability: understandings and misunderstandings. Issues Compr Pediatr Nurs. 1999;22(4):183-196. http://www.ncbi.nlm.nih.gov/pubmed/10827606.

12. Job J. Factors involved in the ineffective dissemination of sexuality information to individuals who are deaf or hard of hearing. Am Ann Deaf. 2004;149(3):264-273.

http://www.ncbi.nlm.nih.gov/pubmed/15552337.

13. França ISX de, Pagliuca LMF. Inclusão social da pessoa com deficiência: conquistas, desafios e implicações para a enfermagem. Rev da Esc Enferm da USP. 2009;43(1):178-185. doi:10.1590/S0080-62342009000100023.

14. Camargo EP de, Nardi R. Dificuldades e alternativas encontradas por licenciandos para o planejamento de atividades de ensino de óptica para alunos com deficiência visual. Rev. Bras. Ensino Física. 2007;29(1):115-126. doi:10.1590/S1806-11172007000100018.

15. Squarcini CFR, Esteves AM. Cronobiologia e inclusão educacional de Pessoas Cegas: do biológico ao social. Rev Bras Educ Espec. 2013;19(4):519-530.

16. Lefèvre F, Lefèvre AMC. O Discurso Do Sujeito Coletivo: Um Novo Enfoque Em Pesquisa Qualitativa (Desdobramentos). 2nd ed. Caxias do Sul: Ed. Diálogo; 2003.

17. Menezes AB de C, Brito RCS. Reflexão sobre a homossexualidade como subproduto da evolução do prazer. Psicol em Estud. 2007;12(1):133-139. doi:10.1590/S1413-73722007000100016.

18. Paiva $V$, Aranha F, Bastos $F$. Opiniões e atitudes em relação à sexualidade: pesquisa de âmbito nacional, Brasil 2005. Rev Saúde Pública. 2008;42(Supl 1):54-64. http://www.scielo.br/pdf/rsp/v42s1/08.pdf.

Accessed April 6, 2014.

19. López-Jaime P, Santos-Ortiz MDC, Dávila-Torres RR, Torres L, Díaz L. Conocimiento en Sexualidad y Prácticas Sexuales en Estudiantes Universitarios con Impedimentos Visuales- Necesidad de Materiales Educativos. P R Health Sci J. 2001;20(3):260-275. http://prhsj.rcm.upr.edu/index.php/prhsj/article/view/1048/688.

20. Barbosa GOL, Wanderley LD, Rebouças CBDA, Oliveira PMP De, Pagliuca LMF. [Development of assistive technology for the visually impaired: use of the male condom]. Rev Esc Enferm USP. 2013;47(5):1163-1169. doi:10.1590/S0080-623420130000500021.

\section{Nota}

1 - Informações extraídas de http://www.icbcuberaba.org.br/ 